\title{
The Micromechanics of Westerley Granite at Large Compressive Loads
}

\author{
H. S. Внат, ${ }^{1,2}$ C. G. SAmmis, ${ }^{1}$ and A. J. Rosakis ${ }^{2}$
}

\begin{abstract}
The micromechanical damage mechanics formulated by AshBY and SAmmis (Pure Appl Geophys 133(3) 489-521, 1990) has been shown to give an adequate description of the triaxial failure surface for a wide variety of rocks at low confining pressure. However, it does not produce the large negative curvature in the failure surface observed in Westerly granite at high confining pressure. We show that this discrepancy between theory and data is not caused by the two most basic simplifying assumptions in the damage model: (1) that all the initial flaws are the same size or (2) that they all have the same orientation relative to the largest compressive stress. We also show that the stress-strain curve calculated from the strain energy density significantly underestimates the nonlinear strain near failure in Westerly granite. Both the observed curvature in the failure surface and the nonlinear strain at failure observed in Westerly granite can be quantitatively fit using a simple bi-mineral model in which the feldspar grains have a lower flow stress than do the quartz grains. The conclusion is that nonlinearity in the failure surface and stress-strain curves observed in triaxial experiments on Westerly granite at low loading rates is probably due to low-temperature dislocation flow and not simplifying assumptions in the damage mechanics. The important implication is that discrepancies between experiment and theory should decrease with increased loading rates, and therefore, the micromechanical damage mechanics, as formulated, can be expected to give an adequate description of high strain-rate phenomena like earthquake rupture, underground explosions, and meteorite impact.
\end{abstract}

\section{Introduction}

The brittle deformation of rock is known to be sensitive to the size and density of internal fractures, which are commonly characterized as damage. The effects of damage are especially important in geomechanical models of phenomena that involve high levels of stress such as earthquakes, underground

1 Department of Earth Sciences, 3651 Trousdale Parkway, Los Angeles, CA 90089, USA. E-mail: hbhat@usc.edu

2 Graduate Aerospace Laboratories, 1200 E. California Blvd., Pasadena, CA 91125, USA. explosions, and meteorite impacts. In such applications the evolution of damage is sensitive not only to the initial damage, but also to the rate at which the load is applied, which is typically very high in these phenomena. The evolution of fracture damage and corresponding changes the state of stress and strain can be modeled using damage mechanics. Most damage mechanics formulations may be characterized as either a continuum model or a micromechanical model. Continuum damage models represent the strain energy as an expansion of the stress invariants and define a damage parameter that follows an evolution law based on the stress. Stress-strain relations are obtained by differentiating the elastic strain energy. Micromechanical formulations model the nucleation, growth, and interaction of the individual cracks. The strain energy in these models is derived using fracture mechanics, which is then differentiated to derive the stress-strain relations.

In this paper, we explore a micromechanical damage mechanics originally formulated by AsHBY and Sammis (1990) and extended by Deshrande and Evans (2008), which is based on the growth and interaction of tensile wing cracks nucleated at the tips of an initial distribution of microcracks. This model incorporates results from many studies of mode I wing cracks nucleated and driven by mode II sliding [for example see Kachanov (1982a, b), Nematnasser and Hori (1982), Ashby and Hallam Née CoOKsley (1986), Jeyakumaran and Rudnicki (1995) and references therein]. By approximating the interaction between growing wing cracks AsHBY and SAmmis (1990) found a positive feedback that led to mechanical instability and failure. They demonstrated that their model gave an adequate description of the failure envelope ( $\sigma_{1}$ vs. $\sigma_{3}$ at failure) for a wide range of rocks loaded in triaxial compression $\left(\sigma_{1}<\sigma_{2}=\sigma_{3}\right.$, where compression is taken as negative). However, as 
illustrated in Fig. 1 for Westerly granite, their model predicts a nearly linear increase the axial stress $\sigma_{1}$ at failure while the triaxial data show large negative curvature, particularly at high values of the confining stress $\sigma_{2}=\sigma_{3}$. Ashby and SAmmis (1990) hypothesized that this curvature is due to a gradual transition to plastic yielding based partly on their observation that the curve is asymptotic to the measured yield stress of quartz, and partly on their observation of a similar transition in the failure envelope of rocks such as marble, limestone, and halite that are known to deform plastically at modest confining stress.

The main objective of this paper is to verify that this observed curvature is indeed due to the onset of plastic flow, and not caused by some physical aspect of the brittle damage accumulation that was lost due to simplifications in the AshBY and SAmmis (1990) model. If the curvature is due to the onset of plasticity, then it should not occur at the very high loading rates that characterize earthquake ruptures, explosions, and impacts. In that case, the more linear micromechanical damage mechanics should give an adequate description of these phenomena to very high

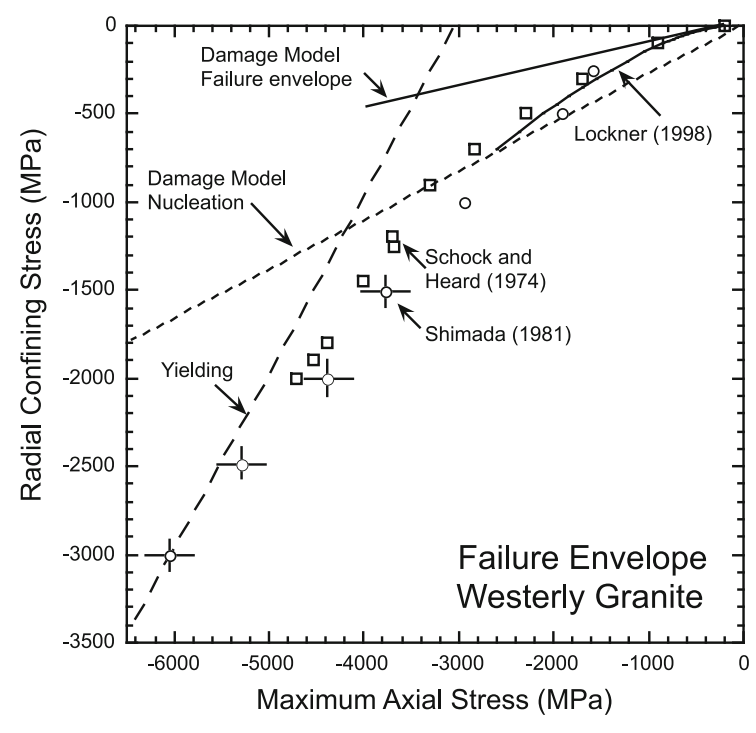

Figure 1

Comparison between failure surface predicted by AsHBY and SAmmis (1990) and experimental observations for granite. The model predicts the linear failure surface well for radial stresses up to $100 \mathrm{MPa}$. At higher confining pressures the measured axial stress at failure is lower than that predicted and eventually approaches the yield surface for granite stresses. However, if the linear failure surface predicted by the damage mechanics is a consequence of one or more of the simplifying assumptions in the Ashby and SAmmis (1990) formulation, then the damage mechanics must be improved before it can be used to model high-stress phenomena.

In this paper we test whether the relaxation of two key assumptions in the Ashby and SAmmis (1990) formulation leads to a model that produces the observed curvature in the failure surface. The first is the assumption that all the starter flaws have the same orientation relative to the largest principal stress $\sigma_{1}$ and the second is that they are all the same size. Physically, these assumptions imply that the nucleation and growth of new damage is controlled by one dominant set of initial microcracks. While this appears to be an adequate approximation at low confining stress, it is possible that high confining stress will suppress the growth of the dominant wing cracks thereby allowing wing cracks to nucleate and grow from smaller and or less favorably oriented starter cracks. This activation of additional sources of damage could result in failure at a lower value of $\sigma_{1}$, and hence produce the observed curvature in the failure surface.

While Ashby and SAmmis (1990) limited their analysis to the failure surface, DeshPANDE and Evans (2008) extended their model by calculating the change in strain energy associated with the damage and using it to calculate strain as a function of loading stress. In this work, we calculate the strain energy associated with the growing wing cracks in a less approximate way than that used by DeshPANDE and Evans (2008), and compare the resultant stress-strain curves with those measured for Westerly granite.

Even with these physically motivated modifications in the assumed size and orientation of the starter flaws and an improved formulation of the strain energy density, we found that the Ashby and SAmmis (1990) brittle damage mechanics alone cannot explain quasistatic triaxial deformation data for Westerly granite. Large observed nonlinearities in the failure envelope ( $\sigma_{1}$ vs. $\sigma_{3}$ at failure) and the stressstrain curve ( $\epsilon_{1}$ vs. $\sigma_{1}$ ) are not predicted by the brittle damage model. However, we show that these nonlinearities can be modeled as resulting from the interaction between low-temperature ductile flow of 
the weak minerals and brittle damage in the strong ones.

\section{Damage Mechanics in Triaxial Compression:} Case 1-The Quasistatic Regime With a Single Flaw Size

Following Ashby and SAmmis (1990) and Deshrande and Evans (2008) we begin by considering an isotropic elastic solid that contains an array of penny shaped cracks all of radius $a$ and all aligned at an angle $\Psi$ to the largest (most negative) remote compressive stress $\sigma_{1}$ (Fig. 2). In order to simulate the radial confining stress in triaxial experiments, we assume $\sigma_{2}=\sigma_{3}$ where $\sigma_{3}$ is the minimum principal remote stress. The size and density of the initial flaws are characterized by an initial damage defined as

$$
D_{o}=\frac{4}{3} \pi N_{V}(\alpha a)^{3}
$$

where $N_{V}$ is the crack density per unit volume and $\alpha$ $a$ is the projection of the crack radius in a vertical plane. Since our goal is to test the damage mechanics against triaxial deformation data for Westerly granite, the loading rate in those experiments is sufficiently slow that dynamic crack growth effects can be ignored.

As in Fig. 2, the remote stresses create a shear stress $\tau$ and a normal stress $\sigma_{n}$ on each penny shaped crack given by

$$
\begin{aligned}
\tau & =\frac{\sigma_{3}-\sigma_{1}}{2} \sin 2 \Psi \\
\sigma_{n} & =\frac{\sigma_{3}+\sigma_{1}}{2}+\frac{\sigma_{3}-\sigma_{1}}{2} \cos 2 \Psi
\end{aligned}
$$

Sliding on the cracks is controlled by a coefficient of friction $\mu$. In the quasistatic case we ignore the difference between static and dynamic friction. For the remainder of this paper we assume that $\Psi=45^{\circ}$ and, hence, $\alpha=0.707$.

Three deformation regimes can be identified based on the value of $\mu$ and the magnitudes of $\tau$ and $\sigma_{n}$ (or equivalently $\sigma_{1}$ and $\sigma_{3}$ ). In order of increasing axial stress they are: (1) initial cracks do not slide, (2) initial cracks slide but wing cracks do not nucleate, and (3) wing cracks nucleate and grow from the tips of the initial inclined cracks. We discuss each in turn.

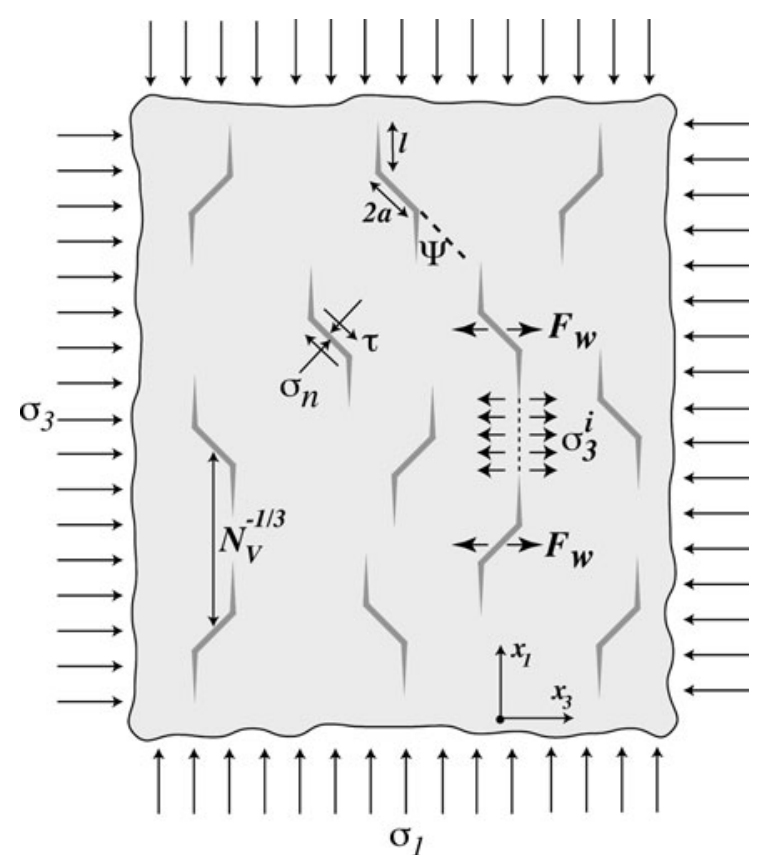

Figure 2

Geometry in the AsHBY and SAMMIS (1990) micromechanical damage mechanics model. Sliding on an array of penny-shaped cracks having volume density of $N_{V}$ and radius $a$ produces a wedging force $F_{w}$ that drives tensile wing cracks to open in the direction of the smallest principal stress $\sigma_{3}$ and propagate parallel to the largest principal stress $\sigma_{1}$. Growth of wing cracks is enhanced by $\sigma_{1}$, retarded by $\sigma_{3}$, and enhanced by a global interaction that produces a mean tensile stress $\sigma_{3}^{i}$. The positive feedback provided by this tensile interaction stress leads to a runaway growth of the wing cracks and ultimate macroscopic failure

\subsection{Regime 1: Initial Cracks Do Not Slide}

If $|\tau| \leq \mu\left|\sigma_{n}\right|$, the initial cracks do not slide and the material deforms as if the cracks were not present. The stress-strain curve is simply $\sigma_{1}=E_{o} \epsilon_{1}$ where $E_{o}$ is the Young's modulus of the undamaged solid. In terms of the principal stresses the condition for regime 1 is $\sigma_{1} \geq[(1+\mu) /(1-\mu)] \sigma_{3}$ (where compression is negative).

\subsection{Regime 2: Initial Cracks Slide But Wing Cracks Do Not Nucleate}

If, $\sigma_{1}<[(1+\mu) /(1-\mu)] \sigma_{3}$ the initial cracks slide, but wing cracks will not nucleate until $\sigma_{1}$ reaches a threshold $\sigma_{1 c}$ given by Ashby and Sammis (1990) as 


$$
\begin{array}{r}
\sigma_{1 c}=\left(\frac{\sqrt{1+\mu^{2}}+\mu}{\sqrt{1+\mu^{2}}-\mu}\right) \sigma_{3}- \\
\left(\frac{\sqrt{3}}{\sqrt{1+\mu^{2}}-\mu}\right) \frac{K_{I C}}{\sqrt{\pi a}}
\end{array}
$$

where $K_{I C}$ is the critical stress intensity factor for quasistatic mode I loading. For axial stresses less compressive (less negative) than this nucleation threshold, sliding on the initial cracks produces a stress concentration at their tips. For a circular crack loaded in shear the loading varies between mode II and mode III around the periphery (Fig. 3). At most locations it is mixed mode and the energy release rate is given by TADA et al. (1985) as

$$
\begin{aligned}
G & =G_{I I}+G_{I I I}=\frac{K_{I I}^{2}}{E_{o}}+\frac{K_{I I}^{2}}{E_{o}} \\
\text { where } K_{I I} & =\frac{4}{\pi(2-v)}\left(\sigma_{s} \cos \phi\right) \sqrt{\pi a} \\
\text { and } K_{I I I} & =\frac{4(1-v)}{\pi(2-v)}\left(\sigma_{s} \sin \phi\right) \sqrt{\pi a}
\end{aligned}
$$

Note that slip is pure mode II at $\phi=0$ and $\pi$ and pure mode III at $\phi=\pi / 2$ and $3 \pi / 2$.

In these expressions, the effective shear stress on the cracks is $\sigma_{s}=\tau+\mu \sigma_{n}$ where $\tau$ and $\sigma_{n}$ are the shear and normal stresses resolved on the crack given by Eq. 2 . The strain energy density can be written as
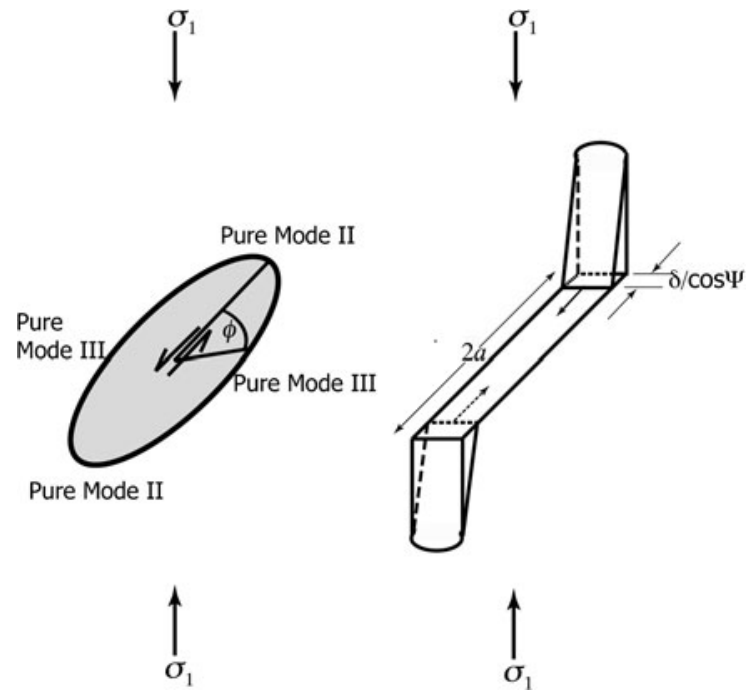

Figure 3

Circular crack under uniaxial loading and wing crack geometry

$$
W=W_{o}+N_{V} \Delta W_{1}
$$

where $W_{o}$ is the elastic contribution, $N_{V}$ is the number of cracks per unit volume, and $\Delta W_{1}$ is the change in elastic strain energy for each crack that is given by

$$
\Delta W_{1}=\frac{2 \pi}{E_{o}} \int_{o}^{a}\left\langle K_{I I}^{2}+K_{I I I}^{2}\right\rangle r d r
$$

It is shown in Appendix A that Eq. 6 can be written

$$
\Delta W_{1}=\frac{16 a^{3} \sigma_{s}^{2}}{3 E_{o}}\left[\frac{1+(1-v)^{2}}{(2-v)^{2}}\right]
$$

and that the corresponding axial strain is

$$
\begin{array}{r}
\epsilon_{1}=\frac{\partial W}{\partial \sigma_{1}}=\frac{\sigma_{1}}{E_{o}}+\frac{2 D_{o}}{\pi \alpha^{3} E_{o}}(1-\mu)^{2} \times \\
{\left[\frac{1+(1-v)^{2}}{(2-v)^{2}}\right]\left[\sigma_{1}-\frac{(1+\mu)}{(1-\mu)} \sigma_{3}\right]}
\end{array}
$$

For uniaxial loading $\sigma_{3}=0$ and thus the effective Young's modulus $E$ is

$$
E=E_{o}\left\{1+\frac{2 D_{o}(1-\mu)^{2}}{\pi \alpha^{3}}\left[\frac{1+(1-v)^{2}}{(2-v)^{2}}\right]\right\}^{-1}
$$

Equation 9 gives the reduction in modulus associated with the initial cracks which can be compared with the damaged modulus calculated using the self-consistent approach by O'CONNELL and BUDIANSKY (1974) for the case $\mu=0$. Note that there is no reduction in modulus until sliding commences at $\sigma_{1} \geq[(1+\mu) /(1-\mu)] \sigma_{3}$.

\subsection{Regime 3: Wing Cracks Nucleate and Grow From The Tips of The Initial Cracks}

If $\sigma_{1}$ is more compressive than the nucleation threshold in Eq. 3, then wing cracks grow from the tips of the initial angle cracks as in Fig. 2. The length of the wing cracks $l$ is described in terms of damage $D$ defined as

$$
D=\frac{4}{3} \pi N_{V}(l+\alpha a)^{3}
$$

When $l=0$ Eq. 10 gives the initial damage $D_{o}$ defined in Eq. 1.

As in Ashby and Sammis (1990) we calculate the mode I stress intensity factor at the tips of the wing 
cracks by approximating an angle crack and its wing cracks as a single tensile crack of radius $l+\alpha a$ that is being wedged open by sliding on the angle crack. The loading stresses create this "wedging force" in the $x_{3}$ direction given by

$$
\begin{aligned}
F_{w} & =\left(\tau+\mu \sigma_{n}\right) \pi a^{2} \sin \Psi \\
& =-\left(A_{1} \sigma_{1}-A_{3} \sigma_{3}\right) a^{2}
\end{aligned}
$$

where the constants $A_{1}$ and $A_{3}$ will be determined subsequently.

The force $F_{w}$ acting at the midpoint of the inclined cracks produces a mode I stress intensity at the tips of their wing cracks (TADA et al., 1985) given by the first term in the equation

$$
K_{I}=\frac{F_{w}}{[\pi(l+\beta a)]^{3 / 2}}+\frac{2}{\pi} \sigma_{3} \sqrt{\pi l}
$$

The second term is the reduction in $K_{I}$ caused by the direct application of $\sigma_{3}$ to the wing cracks. By comparing the equivalent expression for $K_{I}$ in 2D with analytic and numerical results, AsHBY and SAmmis (1990) found they had to introduce a constant $\beta=0.1$ to obtain agreement at small values of $l$ where their mid-point wedging approximation is poor. Agreement at nucleation $(l=0)$ and at large $l$ constrains $A_{1}$ and $A_{3}$ in Eq. 11.

$$
\begin{aligned}
& A_{1}=\pi \sqrt{\frac{\beta}{3}}\left[\sqrt{1+\mu^{2}}+\mu\right] \\
& A_{3}=A_{1}\left[\frac{\sqrt{1+\mu^{2}}+\mu}{\sqrt{1+\mu^{2}}-\mu}\right]
\end{aligned}
$$

where, again, we have assumed that $\Psi=45^{\circ}$. Following Ashby and SAmmis (1990) we further assume that these equations derived in $2 \mathrm{D}$ also hold for penny shaped cracks in $3 \mathrm{D}$, but we adjust $\beta$ to fit experimental data.

The crux of this micromechanical damage mechanics is the interaction between the growing wing cracks. AshBY and SAmmis (1990) estimated this in a global sense by requiring that the net wedging force $F_{w}$ across any vertical section be balanced by an internal stress $\sigma_{3}^{i}$ of the form

$$
\sigma_{3}^{i}=\frac{F_{w}}{\Pi-\pi(l+\alpha a)^{2}}
$$

where $\Pi$ is the average associated with each crack in the array of $N_{V}$ cracks per unit volume. Assigning a spherical volume to each crack gives a maximum circular cross section of

$$
\Pi=\pi^{1 / 3}\left(\frac{3}{4 N_{V}}\right)^{2 / 3}
$$

Hence, as the area of a growing crack approaches its maximum area, the interaction stress increases. Since $\sigma_{3}^{i}$ increases the stress intensity factor on the growing cracks it provides a positive feedback that leads to failure. The total stress intensity factor including the interaction is

$$
K_{I}=\frac{F_{w}}{[\pi(l+\beta a)]^{3 / 2}}+\frac{2}{\pi}\left(\sigma_{3}+\sigma_{3}^{i}\right) \sqrt{\pi l}
$$

Note that in this expression $F_{w}$ and $\sigma_{3}^{i}$ are positive and increase $K_{I}$ while $\sigma_{3}$ is negative and decreases $K_{I}$. In terms of damage, $K_{I}$ can be written

$$
\frac{K_{I}}{\sqrt{\pi a}}=\left(\sigma_{3} A_{3}-\sigma_{1} A_{1}\right)\left(c_{1}+c_{2}\right)+\sigma_{3} c_{3}
$$

where

$$
\begin{aligned}
c_{1} & =\frac{1}{\pi^{2} \alpha^{3 / 2}\left[\left(D / D_{o}\right)^{1 / 3}-1+\beta / \alpha\right]^{3 / 2}} \\
c_{2} & =\frac{2}{\pi} \sqrt{\alpha}\left[\left(D / D_{o}\right)^{1 / 3}-1\right]^{1 / 2} \\
c_{3} & =\frac{2}{\pi^{2} \alpha^{3 / 2}}\left[\left(D / D_{o}\right)^{1 / 3}-1\right]^{1 / 2} \times \\
& {\left[\frac{D_{o}^{2 / 3}}{1-D^{2 / 3}}\right] }
\end{aligned}
$$

These expressions correct an algebra error common to Deshrande and Evans (2008) and Ashby and SAmmis (1990) where a factor of $\beta / \alpha$ was dropped in deriving Eq. 26 in the latter.

At this point in their development, Deshpande and Evans (2008) assume $\sigma_{2}=\left(\sigma_{1}+\sigma_{3}\right) / 2$ and express their results in terms of the mean stress $\sigma_{m}=\left(\sigma_{1}+\right.$ $\left.\sigma_{3}\right) / 2$ and von-Mises effective stress $\sigma_{e}=\sqrt{3}\left(\sigma_{3}-\right.$ $\left.\sigma_{1}\right) / 2$ in order to facilitate the transition to plastic yielding at very high stresses. In this case the stress intensity factor can be written

$$
\frac{K_{I}}{\sqrt{\pi a}}=A \sigma_{m}+B \sigma_{e}
$$


where the corrected expressions for $A$ and $B$ are

$$
\begin{aligned}
& A=\left(A_{3}-A_{1}\right)\left(c_{1}+c_{2}\right)+c_{3} \\
& B=\frac{1}{\sqrt{3}}\left[\left(A_{3}+A_{1}\right)\left(c_{1}+c_{2}\right)+c_{3}\right]
\end{aligned}
$$

However, we choose to continue our development in terms of $\sigma_{1}$ and $\sigma_{3}$ because we wish to test the results against triaxial laboratory data for which the stress state is an axial load $\sigma_{1}$ and a radial confining pressure $\sigma_{2}=\sigma_{3}$.

During quasistatic triaxial experiments the confining stress $P_{c}=\sigma_{2}=\sigma_{3}$ is fixed while the axial load begins at $\sigma_{1}=\sigma_{3}$ and is slowly increased to macroscopic failure. Once the nucleation condition is reached, further increases in $\sigma_{1}$ cause the wing cracks to grow. For each increase in $\sigma_{1}$, the wing cracks grow until $K_{I}$ falls to its critical value $K_{I C}$, which is a material property. For most oxides and silicates $K_{I C}=1 \mathrm{MPa} \mathrm{m}^{1 / 2}$. Setting $K_{I}=K_{I C}$ in Eq. 17 gives the following relation between the principal stresses $\sigma_{1}, \sigma_{3}$ and the equilibrium damage $D$ at failure,

$$
S_{1}=\frac{S_{3}\left[c_{3}+A_{3}\left(c_{1}+c_{2}\right)\right]-1}{A_{1}\left(c_{1}+c_{2}\right)}
$$

where the dimensionless principal stresses are defined as $S_{j}=\sigma_{j} \sqrt{\pi a} / K_{I C}$.

For a fixed value of $S_{3}$, the dimensionless axial stress $S_{1}$ increases with increasing equilibrium damage to a maximum value beyond which it decreases with increasing equilibrium damage leading to an instability and macroscopic failure. The maximum in the plot of $S_{1}$ versus damage is the failure stress.

Ashby and Sammis (1990) fit this model to the experimental failure envelopes of a number of brittle solids using the following procedure. First, the nucleation equation was fit to the stress at which new damage commenced (as indicated by the first nonlinearity in the stress-strain curve or by the onset of acoustic emissions). This determined the coefficient of friction $\mu$ on the starter flaws and their radius a. Note that the density of starter flaws does not appear in the nucleation equation because they are assumed not to interact at nucleation. Having determined $\mu$ and $a$, the triaxial failure envelope ( $\sigma_{1}$ vs. $\sigma_{3}$ at failure) was fit (at low confining stress) by varying the parameter $\beta$ and the value of the initial damage $D_{o}$ as discussed further in a subsequent section.
Deshpande and Evans (2008) added a term to the strain energy density in Eq. 5 that represents the increase in elastic energy density associated with a pair of wing cracks each of length $l$

$$
W=W_{o}+N_{V} \Delta W_{1}+N_{V} \Delta W_{2}
$$

where

$$
\Delta W_{2}=\frac{2 \pi}{E_{o}} \int_{\alpha a}^{l+\alpha a} K_{I}^{2} r d r
$$

As before, we approximate the angle starter crack plus the two wing cracks as one effective tensile crack of length $2(l+\alpha a)$. Note that Eq. 23 differs from that used by Deshrande and Evans (2008) who, to simplify the integration, assumed that the wing cracks grow at a fixed value of $l / a$ and wrote

$$
\Delta W_{2}=\frac{2 \pi}{E_{o}} \int_{0}^{a} K_{I}^{2} r d r
$$

Since $K_{I}^{2}$ is a constant for fixed $l / a$ it was removed from the integral giving the simple result

$$
\begin{aligned}
W= & W_{o}+N_{V} \Delta W_{1}+ \\
& \frac{\pi D_{o}}{4 \alpha^{3} G_{o}(1+v)}\left(A \sigma_{m}+B \sigma_{e}\right)^{2}
\end{aligned}
$$

where $G_{o}$ is the undamaged shear modulus and $v$ is Poisson's ratio. However, at this level of approximation the third term on the right hand side is a constant. Since $K_{I}$ is a constant $K_{I}=K_{I C}$ during quasistatic crack growth, the quantity $\left(A \sigma_{m}+B \sigma_{e}\right)$ is also constant (see Eq. 19). The result is that the contribution of the wing cracks to the energy density in Eq. 25 is independent of their size because the stress and length of wing cracks are linked by the requirement that $K_{I}=K_{I C}$. Hence, in their approximation, crack growth produces zero additional strain beyond the elastic strain, when the strain is calculated as $\partial W / \partial \sigma_{i j}$.

However, the recognition that during quasi-static crack growth allows it to be removed from the integral in Eq. 23 giving the simple result.

$$
\Delta W_{2}=\frac{\pi K_{I c}^{2}(\alpha a)^{2}}{E_{o}}\left[\left(\frac{D}{D_{o}}\right)^{2 / 3}-1\right]
$$


and we write the energy density as

$$
\begin{aligned}
W= & W_{o}+N_{V} \Delta W_{1}+ \\
& \frac{3 \pi K_{I c}^{2} D_{o}}{4 E_{o} \alpha a}\left[\left(\frac{D}{D_{o}}\right)^{2 / 3}-1\right]
\end{aligned}
$$

Since $D$ increase with up to failure, the strain contributed by the growing wing cracks (third term on the right in Eq. 27) is no longer zero.

This change does not significantly affect DESHPANDE and Evans (2008) simulations of high-velocity impact where most of the damage occurs in the tensile and ductile regimes. However, it is important at lower stresses.

Once wing cracks nucleate, the mode II stress concentrations at the tips of an initial flaw are replaced by mode I concentrations at the tips of the wing cracks and no longer contribute to $\Delta W_{1}$. However, sliding on the initial flaw continues as $l$ increases, and the mode III stress intensity at the edges of the initial flaw continue to increase. Opening of the wing cracks allows significantly more sliding than that caused by the remote stress (as in the derivation of Eq. 7). As illustrated in Fig. 3, we model the sliding crack terminated by wing cracks as two parallel screw dislocations, each of length $2 a$ and having a Burgers vector $b$ equal to the sliding displacement $\delta$ associated with the wing cracks of length $l$, which is given by JOHNSON and SAmmis (2001) as

$$
\delta=\frac{6}{\sqrt{\pi} \cos \Psi} \frac{(\lambda+2 G)}{G(\lambda+G)} K_{I C} \sqrt{l}
$$

The change in elastic energy per unit length of screw dislocation is $E_{l}=G b^{2}$, so the change in energy for each inclined crack (for $\Psi=45^{\circ}$ ) can be written directly as

$$
\begin{aligned}
\Delta W_{1}= & 4 a G \delta^{2} \\
= & \frac{288}{\pi}\left(\frac{\lambda+2 G}{\lambda+G}\right)^{2} \frac{K_{I C}^{2}}{G} l a \\
= & \frac{288}{\pi}\left(\frac{\lambda+2 G}{\lambda+G}\right)^{2} \\
& \times\left(\frac{K_{I C}^{2}}{G} a^{2} \alpha\right)\left[\left(\frac{D}{D_{o}}\right)^{1 / 3}-1\right]
\end{aligned}
$$

Eq. 27 becomes

$$
\begin{aligned}
& W=W_{o}+\frac{216}{(\alpha \pi)^{2}} \times \\
& \left(\frac{\lambda+2 G}{\lambda+G}\right)^{2}\left(\frac{K_{I C}^{2}}{a G} D_{o}\right) \times \\
& {\left[\left(\frac{D}{D_{o}}\right)^{1 / 3}-1\right]+} \\
& \frac{3}{4 \alpha}\left(\frac{K_{I C}^{2}}{a E_{o}} D_{o}\right)\left[\left(\frac{D}{D_{o}}\right)^{2 / 3}-1\right] .
\end{aligned}
$$

\subsection{Allowing Non-Parallel Initial Flaws}

The formulations of Ashby and SAmmis (1990) and Deshpande and Evans (2008) assume that the growing wing cracks are all parallel. We still assume all starter flaws are at the same angle $\Psi$ with respect to $x_{1}$, but now assume that the components of their normal vectors in the $x_{1}$ and $x_{1}$ directions are randomly distributed as in Fig. 4. Hence, they do not all produce the same tension across a random vertical section as assumed in Eq. 14. This can be corrected by replacing $F_{w}$ in Eq. 14 for the interaction stress with its angularly averaged value $2 F_{w} / \pi$.

Also, the area per crack must be corrected for the orientation of the cracks. This we do by replacing Eq. 15 with

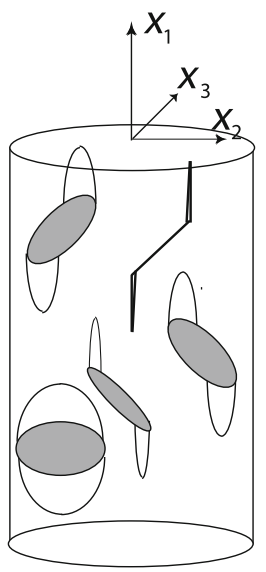

Figure 4

Various flaws having the same orientation with respect to $X_{2}-X_{3}$ plane but rotated about the $X_{1}$ axis 


$$
\Pi=\gamma \pi^{1 / 3}\left(\frac{3}{4 N_{V}}\right)^{2 / 3}
$$

where the adjustable parameter $\gamma \geq 1$ has been introduced to reflect the fact that the wedging force is balanced by a larger fraction of a random cross-section. For example, wing cracks orthogonal to an arbitrary cross-section do not reduce the load bearing area. We evaluate $\gamma$ by fitting the triaxial data.

Both corrections for crack orientation affect only the $c_{3}$ term in Eq. 18, which becomes

$$
\begin{aligned}
c_{3}= & \frac{2}{\pi^{2} \alpha^{3 / 2}}\left[\left(D / D_{o}\right)^{1 / 3}-1\right]^{1 / 2} \times \\
& {\left[\frac{D_{o}^{2 / 3}}{1-D^{2 / 3}}\right] }
\end{aligned}
$$

\subsection{Testing Case 1 Using Deformation Data for Westerly Granite}

Westerly granite is composed of quartz ( 27.5\%), plagioclase feldspar ( $\sim 35.4 \%$ An17), potassium feldspar $(\sim 31.4 \%)$ and small amounts of biotite and other minerals $(\sim 4.9 \%)$. The grain size is uniform with a mean diameter of about $300 \mu \mathrm{m}$ and a very low porosity ( $\ll 1 \%$ ) (BRACE, 1965; HufFMAN et al., 1993). The Young's modulus is $E_{o}=70 \mathrm{GPa}$ and Poisson's ratio is $v=0.25$.

\subsubsection{Fitting the Nucleation Stress}

Ashby and Sammis (1990) show that data for nucleation of wing cracks in Westerly granite based on acoustic emissions is reasonably fit by $\sigma_{1}=$ $3.3 \sigma_{3}+79 \mathrm{MPa}$ which, when evaluated using Eq. 3, gives $\mu=0.63$ and $a=0.5 \mathrm{~mm}$. For these values, the requirement that Eq. 21 yield the observed nucleation stress $\sigma_{1}=79 \mathrm{MPa}$ when $\sigma_{3}=0$ sets $\beta=0.32$.

\subsubsection{Calculating the Failure Envelope}

LOCKNER (1998) measured the failure envelope for Westerly granite under axisymmetric loading $\left(\sigma_{1}>P_{c}, P_{c}=\sigma_{2}=\sigma_{3}\right.$ ) to a confining pressure of $P_{c}=200 \mathrm{MPa}$. Adding measurements from ByerLEE (1967); WAWERSIK (1973), he fit the combined failure envelope (to $P_{c}=700 \mathrm{MPa}$ ) with the relation

$$
\sigma_{1 p}=-8.3+\left(46660.4+5128.7 P_{c}\right)^{1 / 2}+P_{c}
$$

where $\sigma_{1 p}$ is the peak axial stress that the sample can support before failure. Equation 33 is plotted in Fig. 1, where it has been extended to higher values of $P_{c}$ using data from ScHOCK and Heard (1974); Shimada (1981).

Using the values of $\mu, a$ and $\beta$ from the preceding analysis of nucleation, and using from Eq. 32 that includes the interaction correction, we found $D_{o}$ as a function of $\gamma$ such that Eq. 21 yields the observed uniaxial strength $\sigma_{1 p}=208 \mathrm{MPa}$ when $P_{c}=0$.

We then calculated the peak axial stress as a function of $P_{c}$ for values of $\gamma$ ranging from $\gamma=1$ to $\gamma=1.5$ and compared with the experimental data in Fig. 5. Note that the theoretical failure envelope is not sensitive to the choice of $\gamma$. This is because the decrease in net wedging force and increase in loadbearing cross-section due to the distributed orientations are compensated by the larger value of the initial damage that is required to fit the uniaxial strength at $P_{c}=0$.

Note also that the experimental failure envelope in Fig. 5 has more curvature than does the nearly linear theoretical envelope. AshBY and SAmmis (1990)

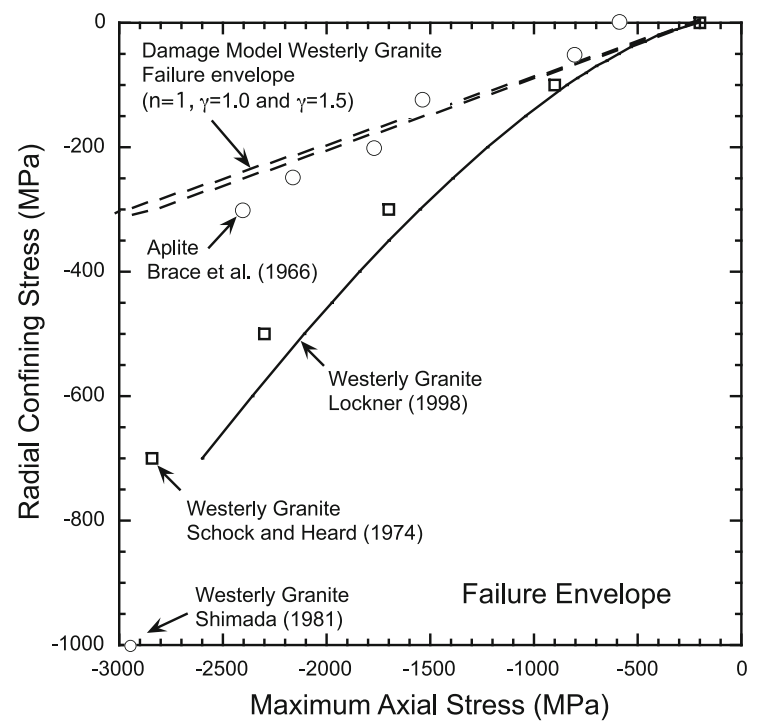

Figure 5

Comparison of the failure envelope for Westerly granite and Aplite with the Damage Mechanics model 
proposed that this might be due to the onset of plastic yielding at high stresses. The yield surface is given by

$$
\sigma_{y}^{2}=\frac{1}{2}\left[\left(\sigma_{1}-\sigma_{2}\right)^{2}+\left(\sigma_{2}-\sigma_{3}\right)^{2}+\left(\sigma_{3}-\sigma_{1}\right)^{2}\right]
$$

where the yield stress can be found from the hardness $H$ since $\sigma_{y}=H / 3$. The yield surface for granite at high stress is plotted as the heavy dashed line in Fig. 1. Note that the transition from brittle failure as represented by the damage mechanics to pure plastic yielding is extremely broad ranging roughly from $P_{c}=100$ to $1,000 \mathrm{MPa}$. This may reflect a range in yield stresses for the different minerals in granite, or it may reflect a range of starter flaw sizes. In the latter case, growth of wing cracks from larger flaws is suppressed at high values of the confining stress giving the myriad of smaller ones a chance to nucleate and grow wing cracks before failure. We investigate both possibilities in subsequent sections.

\subsubsection{Calculating the Stress-Strain Curve}

We used Eqs. 21 and 30 to calculate $\sigma_{1}$ and $W$ as a function of damage, and then calculated the strain as

$$
\epsilon_{1}=\frac{\partial W}{\partial \sigma_{1}}=\frac{\partial W / \partial D}{\partial \sigma_{1} / \partial D}
$$

The case $P_{c}=200 \mathrm{MPa}$ is shown in Fig. 6 where the damage calculations are compared with experimental data reported by LOCKNER (1998). Note that the nonlinear axial strain associated with opening the wing cracks is an order of magnitude less than that due to sliding on the initial flaws. Note also that the nonlinear strain at failure for the random orientation of starter flaws $(\gamma=1.5$ in Fig. 6b) is about twice that for the aligned flaws $(\gamma=1.0$ in Fig. 6a). However, the calculated axial strain at failure in both cases is a factor of 2-4 times less than observed.

The estimate of nonlinear strain given by differentiating Eq. 30 can be tested using an independent estimate that uses the moment tensors of the individual flaws as described by Kostrov (1974) (see Appendix B). The result (for $\Psi=45^{\circ}$ ) is

$$
\begin{aligned}
\epsilon_{11} & =\frac{3}{2^{3 / 4}}\left(\frac{\lambda+2 G}{\lambda+G}\right) \times \\
& \frac{K_{I C} D_{o}}{G \sqrt{\pi a}}\left[\left(\frac{D}{D_{o}}\right)^{2 / 3}-1\right]^{1 / 2} \times \\
& \left\{\frac{12}{\sqrt{2}}+\frac{\lambda}{G}\left[\left(\frac{D}{D_{o}}\right)^{2 / 3}-1\right]^{2}\right\}
\end{aligned}
$$

The first term in the curly brackets is the contribution from sliding on the initial flaws while the second term is the contribution from tensile opening of the wing cracks. Each is plotted in Fig. 6a where they are seen to be in rough agreement with estimates based on differentiating the energy Eq. 30

\section{Damage Mechanics in Triaxial Compression: Case 2-The Quasistatic Regime With a Distribution of Flaw Sizes}

The observed curvature in the failure envelope and in the stress strain curve could be caused by a distribution of initial flaw sizes. Increasing confining pressure might suppress the growth of the larger wing cracks allowing the smaller ones to grow before failure. The increased activation of smaller flaws may produce failure at a lower axial stress and more nonlinear strain —as observed. HADLEY (1976) used a scanning electron microscope to measure the distribution of crack lengths in virgin and in previously stressed Westerly granite shown in Fig. 7. The number of cracks increases sharply as the size decreases between about $50-1 \mu \mathrm{m}$. The longest observed crack was $565 \mu \mathrm{m}$. Also included in Fig. 7 are a fractal distribution and a binomial distribution, both of which contain roughly ten times the number of flaws measured by Hadley.

We model HADLEY (1976)'s measurements as a discrete distribution of flaws in which the largest is assigned index 0 . It has radius $a_{o}$ and a volume density $N_{V o}$ flaws $/ \mathrm{m}^{3}$. Smaller flaws in the distribution are indexed $n=1,2,3$, etc. They have radii $a_{n}$ and volume densities $N_{V n}$. We define the initial damage in terms of the largest flaw as 

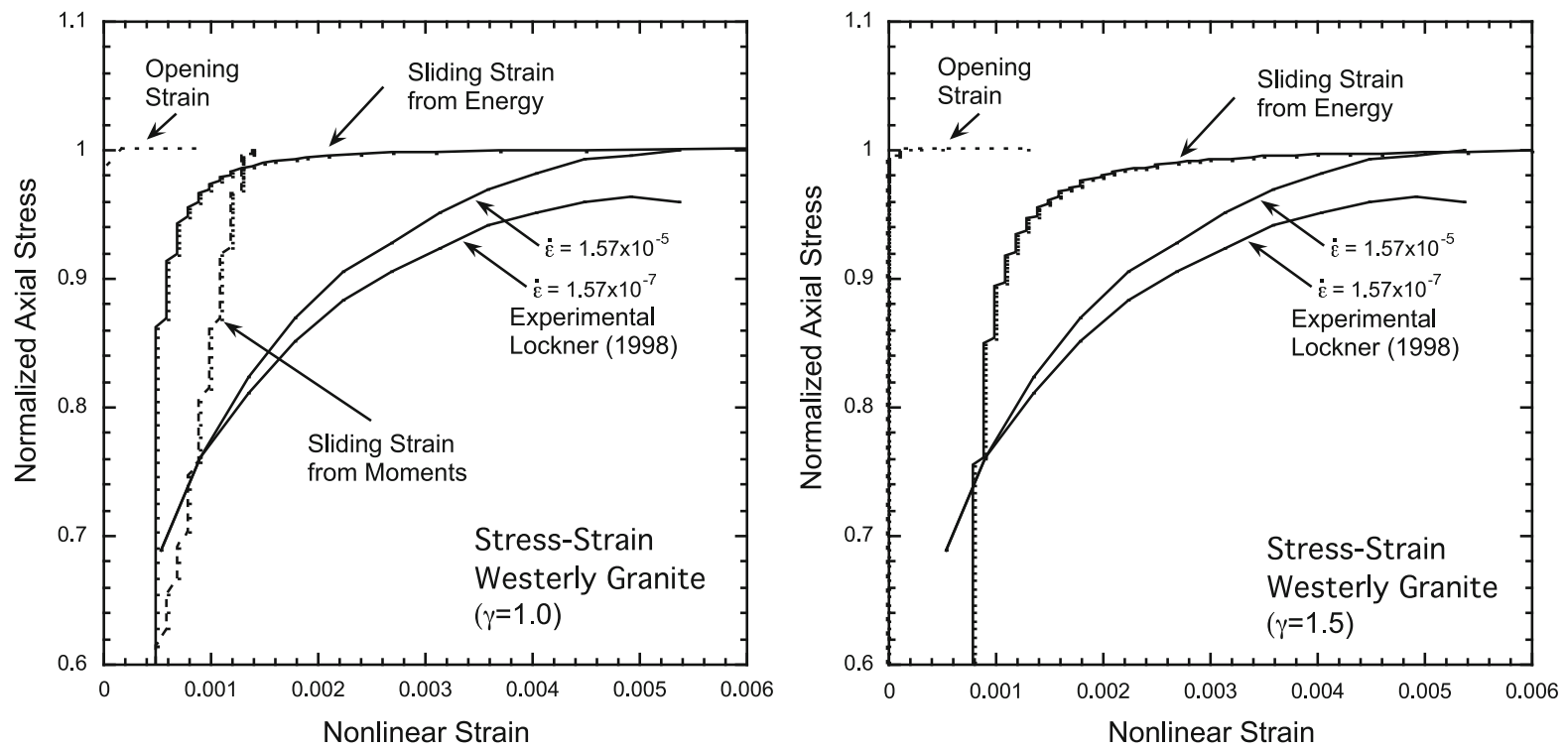

Figure 6

Normalized axial stress versus Nonlinear strain from the Damage Mechanics model for $\gamma=1.0$ and $\gamma=1.5$

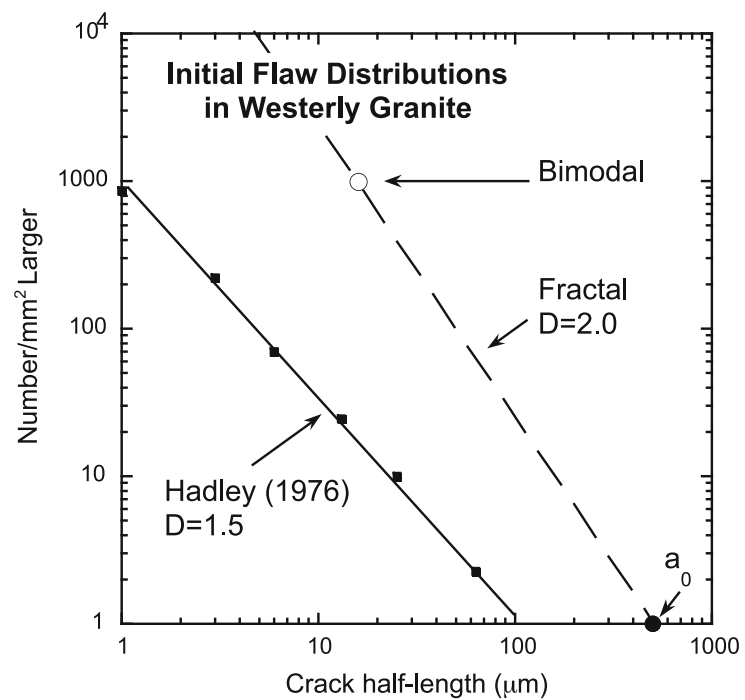

Figure 7

Various flaw size distributions in Westerly granite

$$
D_{o}=\frac{4}{3} \pi N_{V o}\left(\alpha a_{o}\right)^{3}
$$

The wedging force on a class $n$ flaw is given by (see Eq. 11)

$$
F_{w n}=-\left(A_{1} \sigma_{1}-A_{3} \sigma_{3}\right) a_{n}^{2}
$$

and the stress intensity factor at the tips of the wing cracks in each class (ignoring the interaction) is therefore

$$
K_{I n}=\frac{F_{w n}}{\left[\pi\left(l_{n}+\beta a_{n}\right)\right]^{3 / 2}}+\frac{2}{\pi} \sigma_{3} \sqrt{\pi l_{n}}
$$

The interaction term depends again on the wedging force across an average cross-sectional area and the intact area that supports it. We partition space by assigning a spherical volume $4 \pi r^{3} / 3=1 / N_{V o}$ to each of the largest flaws. The area associated with each largest flaw is therefore

$$
\Pi=\gamma \pi^{1 / 3}\left(\frac{3}{4 N_{V o}}\right)^{2 / 3}=\gamma \frac{\pi(\alpha a)^{2}}{D_{o}^{2 / 3}}
$$

where we again include the factor $\gamma \geq 1$ to adjust for random orientation of the largest flaws.

We consider all the cracks as eroding this area and write the equivalent of Eq. 14 as

$$
\sigma_{3}^{i}=\frac{\left(\frac{2}{\pi}\right) \sum_{j=0}^{n}\left(\frac{N_{A j}}{N_{A o}}\right) F_{w j}}{\Pi-\pi \sum_{j=0}^{n}\left(\frac{N_{A j}}{N_{A o}}\right)\left(l_{j}+\alpha a_{j}\right)^{2}}
$$

where the numerator is the sum of the wedging forces on the cross-section belonging to one largest crack and the denominator is the remainder of that crosssection which is supporting the forces. Note that the angular averaging term $2 / \pi$ has again been included. In this expression, $N_{A j}$ is the number of class $j$ particles per area. It is related to the volume density by 
$N_{A j}=2 \alpha a_{j} N_{V j}$. Hence the ratios in Eq. 41 can be written in terms of the volume densities as

$$
\frac{N_{A n}}{N_{A o}}=\left(\frac{a_{n}}{a_{o}}\right) \frac{N_{V n}}{N_{V o}}
$$

and we will specify our discrete flaw distributions using the ratios $a_{n} / a_{o}$ and $N_{V n} / N_{V o}$.

Note that the denominator in Eq. 41 must be positive. For any given distribution of flaw sizes, this imposes an upper bound on $D_{o}$ such that the total area occupied by the flaws in a random cross section can not be larger than the area apportioned to one largest flaw.

$$
\begin{aligned}
\Pi & >\pi \sum_{j=0}^{n}\left(\frac{N_{A j}}{N_{A o}}\right)\left(\alpha a_{j}\right)^{2} \\
& =\pi \sum_{j=0}^{n}\left(\frac{a_{j}}{a_{o}}\right)\left(\frac{N_{V j}}{N_{V o}}\right)\left(\alpha a_{j}\right)^{2}
\end{aligned}
$$

Using Eq. 40 for $\Pi$ we get

$$
D_{o}<\left[\frac{1}{\gamma} \sum_{j=0}^{n}\left(\frac{N_{V j}}{N_{V o}}\right)\left(\frac{a_{j}}{a_{o}}\right)^{3}\right]^{3 / 2}
$$

The stress intensity factor on the wing crack in each size class $n$ becomes

$$
K_{I n}=\frac{F_{w n}}{\left[\pi\left(l_{n}+\beta a_{n}\right)\right]^{3 / 2}}+\frac{2}{\pi}\left(\sigma_{3}+\sigma_{3}^{i}\right) \sqrt{\pi l_{n}}
$$

Our calculation procedure is as follows. We first choose a confining stress $\sigma_{3}$. We then choose an axial stress $\sigma_{1}>\sigma_{3}$. Finally, we use Eqs. 41 and 45 to calculate the set of $l_{n}$ which make $K_{I n}=K_{I C}$ for all size classes $n$ that have nucleated wing cracks. Note that this is an iterative calculation since the interaction term $\sigma_{3}^{i}$ involves all the size classes. Failure is defined as the maximum value of $\sigma_{1}$ for which it is possible to find a set of finite values of the $l_{n}$ for which $K_{I n}=K_{I C}$.

Using the distributions of flaw sizes in Fig. 7, we calculated the failure envelopes plotted in Fig. 8. Note that the incorporation of HADLEY's (1976) measured distribution of smaller microfractures does not produce significant curvature. While it is possible to reduce the failure stress at high confining pressures by incorporating the artificially large distributions of microfractures also shown in Fig. 8, the resultant

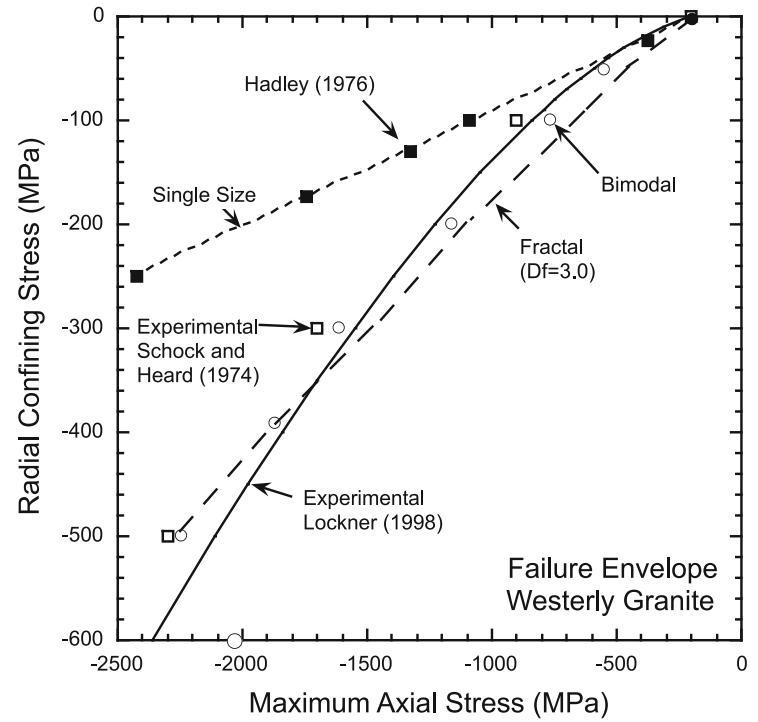

Figure 8

Failure stress curve from the Damage Mechanics model for various flaw size distributions

failure envelope is still linear and a poor fit to LOCKNER's (1998) data. The predicted strain at failure for the Hadley distribution is also indistinguishable from that for a single flaw size and is thus too small by a factor or about four.

\section{Damage Mechanics in Triaxial Compression: Case 3-The Quasistatic Regime in a Multi- Mineralic Rock}

One possible explanation of the observed nonlinearities in failure envelope and the stress-strain curve is that they are produced by different flow stresses in the different minerals that comprise Westerly granite. AshBY and SAmmis (1990) argued that the shape of the failure envelope at very high confining pressures is consistent with plastic flow of the entire rock. It may be that plastic flow (induced due to dislocation pile-ups, intra-granular cracking, void collapse among various mechanisms for rock like materials) in the weaker minerals at lower stresses produces the observed broad transition to pure plasticity. We explore this possibility using the simple bi-mineral model in Fig. 9. The stronger mineral (quartz) is represented by an elastic element with Young's modulus $E_{1}$ in series with a nonlinear 
Table 1

Material properties for westerly granite

\begin{tabular}{|c|c|c|c|}
\hline Material property & Symbol & Value & Units \\
\hline Quartz fraction in Westerly granite & $\xi$ & 0.275 & - \\
\hline Young's modulus for Westerly granite & $E$ & 70 & $\mathrm{GPa}$ \\
\hline Young's modulus for $\alpha$-quartz & $E_{1}$ & 96 & $\mathrm{GPa}$ \\
\hline Young's modulus for feldspar & $E_{2}$ & 61.3 & $\mathrm{GPa}$ \\
\hline Poisson's ratio & $v$ & 0.25 & - \\
\hline Coefficient of friction on microcracks & $\mu$ & 0.63 & - \\
\hline Radius of dominant initial flaws in granite & $a$ & $0.55 \times 10^{-3}$ & $\mathrm{~m}$ \\
\hline Burgers vector for quartz $\mathrm{z}^{\mathrm{a}}$ & $b_{1}$ & $5.2 \times 10^{-10}$ & $\mathrm{~m}$ \\
\hline Burgers vector for Feldspar ${ }^{\mathrm{b}}$ & $b_{2}$ & $9.4 \times 10^{-10}$ & $\mathrm{~m}$ \\
\hline Flow stress in quartz & $\sigma_{o 1}$ & -7.8 & $\mathrm{GPa}$ \\
\hline Flow stress in feldspar & $\sigma_{o 2}$ & -0.78 & $\mathrm{GPa}$ \\
\hline Lattice resistance/modulus: (quartz) ${ }^{\mathrm{c}}$ & $\hat{\tau}_{p} / G$ & $8.9 \times 10^{-1}$ & - \\
\hline Lattice resistance/modulus: (feldspar) ${ }^{\mathrm{c}}$ & $\hat{\tau}_{p} / G$ & $4.5 \times 10^{-2}$ & - \\
\hline Pre-exponential for lattice resistance (both) & $\dot{\epsilon}_{p}$ & $10^{11}$ & $\mathrm{~s}^{-1}$ \\
\hline Activation energy for lattice resistance (both) & ${ }_{p} / G b^{3}$ & 0.05 & - \\
\hline
\end{tabular}

${ }^{\text {a Avg. a, c }}$

b Avg. a, b, c

c Adjusted to give flow stress in Fig. 12

element to be described shortly. The weaker mineral (representing some average of the feldspars) is modeled as an elastic element with lower modulus $E_{2}$ in series with a softer nonlinear element. Values for $E_{1}$ and $E_{2}$ from BAss (1995) are given in Table 1. Triaxial experiments are simulated by loading both minerals in parallel at a constant strain-rate, $\dot{\epsilon}_{o}$.

The observed stress $\bar{\sigma}$ is an average of the stress $\sigma_{1}$ supported by the quartz and $\sigma_{2}$ by the feldspars weighted by their area fractions ( $\xi$ for quartz and $1-\xi$ for the feldspars)

$$
\bar{\sigma}=\xi \sigma_{1}+(1-\xi) \sigma_{2}
$$

The strain rate in each mineral $(i=1,2)$ is a combination of elastic and nonlinear deformation which, for each mineral, add up to the imposed strain rate $\dot{\epsilon}_{o}$.

$$
\dot{\epsilon}_{i e l}+\dot{\epsilon}_{i n l}=\dot{\epsilon}_{o}
$$

The elastic strain rate in each element is $\dot{\epsilon}_{i e l}=\dot{\sigma}_{i} / E_{i}$.

The nonlinear flow at room temperature is accommodated by dislocation glide, which is rate limited by either lattice resistance or obstacles (such as impurities, precipitates, or other dislocations). Since both must be overcome, the one with the lowest strain rate controls flow. At room temperature in quartz and feldspar's, lattice resistance is rate

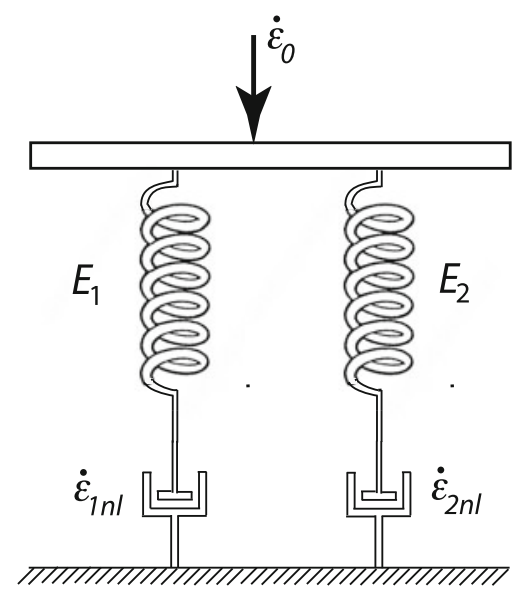

Figure 9

Bi-mineralic model setup for Westerly granite

limiting; its flow law is given by AsHBY and VerRALL (1977) as

$$
\begin{aligned}
\dot{\epsilon}_{\text {inl }} & =\dot{\epsilon}_{i p}\left(\frac{\sigma_{s i}}{G_{i}}\right)^{2} \times \\
& \exp \left\{-\frac{\Delta F_{i p}}{k T}\left[1-\left(\frac{\sigma_{s i}}{\hat{\tau_{i p}}}\right)^{\frac{3}{4}}\right]^{\frac{4}{3}}\right\}
\end{aligned}
$$

where $\sigma_{s i}=\sigma_{i}-P_{c}$ is the shear stress in each element and $P_{c}$ is the confining pressure. Although the 
parameters in Eq. 48 are not known for either quartz or feldspar, they are estimated for feldspar in Table 1 based on the values and scaling relations given by Ashby and Verrall (1977). Equation 48 is plotted in Fig. 10 using these estimated parameters. Note that the strain-rate is very sensitive to the stress. Because the relation is a power law, an order of magnitude increase in strain rate produces a $2.8 \%$ increase in flow stress of the feldspars across a range of more than 20 orders of magnitude in strain-rate. We shall see in the next section that this translates into about a $2 \%$ increase in failure stress for each factor of ten increase in the loading rate, consistent with LOCKNER's (1998) observations. This sensitivity of strain-rate to small changes in stress has the effect of producing a sharp stress threshold for nonlinear deformation and locks the stress at this threshold.

\subsection{Fitting the Multi-Mineral Model to Deformation Data for Westerly Granite}

The objective in this section is to see if observed nonlinearities in failure stress and stress-strain curves can be ascribed to lattice limited flow in the feldspars using estimates of the flow law parameters given in Table 1 . We begin with the failure envelope in Fig. 11a where a horizontal dashed line has been added to Fig. 5 indicating

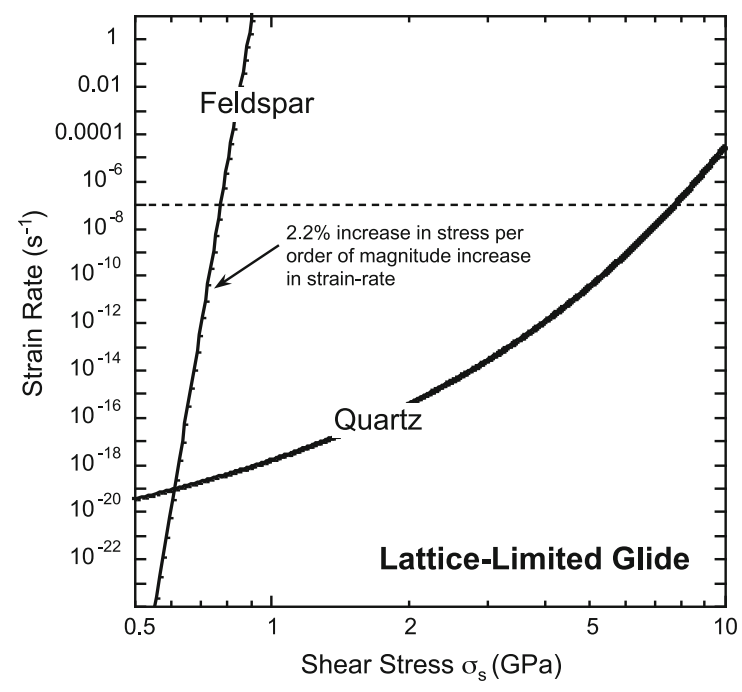

Figure 10

Ashby and Verral (1977) flow rule for Quartz and Feldspar
$P_{c}=-200 \mathrm{MPa}$. We assume that there is no nonlinear flow in quartz at this confining pressure so that it fails at $\sigma_{1}^{*}=-2,000 \mathrm{MPa}$ as predicted by the damage mechanics and indicated by the dashed arrow A. The superscript $*$ is used here to indicate values at failure. Young's modulus for crystalline a-quartz depends on the orientation of loading relative to the crystallographic axes. For elongations along the axes, BASs (1995) gives $c_{11}=86.6 \mathrm{GPa}$, $c_{22}=106.1 \mathrm{GPa}$, and an isotropic average Young's modulus of $E_{1}=96 \mathrm{GPa}$. The total strain in the quartz at failure is entirely elastic, and given by $\epsilon_{o}^{*}=\sigma_{1}^{*} / E_{1}=2.08 \times 10^{-2}$.

The observed failure stress at $P_{c}=-200 \mathrm{MPa}$ is $\bar{\sigma}^{*}=-1230 \mathrm{MPa}$ as indicated by the dashed arrow B. Since quartz comprises $27.5 \%$ of Westerly granite, we choose $\xi=0.275$ and solve Eq. 46 to find $\sigma_{2}^{*}=-938 \mathrm{MPa}$, indicated by the dashed arrow $\mathrm{C}$ in Fig. 11a. Since the model assumes that both minerals are loaded in parallel, the total strain in the feldspars at failure is also $\epsilon_{o}^{*}=2.08 \times 10^{-2}$. However, strain in the feldspars is partly elastic and partly non-elastic. The non-elastic contribution to the strain at failure was measured by LOCKNER (1998) to be about $\epsilon_{2 n l}^{*}=$ $5.5 \times 10^{-3}$ (see Fig. 6). Since we assume that all the nonlinear strain occurs in the feldspar, the elastic strain is $\epsilon_{2 e l}^{*}=\epsilon_{o}^{*}-\epsilon_{2 n l}^{*}=1.53 \times 10^{-2}$, which corresponds to a Young's modulus for the feldspars of $E_{2}=\sigma_{2}^{*} / \epsilon_{2 e l}^{*}=61.3 \mathrm{GPa}-\mathrm{a}$ value consistent with those for feldspars given by BAss (1995). The weighted average of $E_{1}$ and $E_{2}$ also gives a reasonable value of $\bar{E}=70.8 \mathrm{GPa}$ for Westerly granite. Figure $11 \mathrm{~b}$ shows that the failure stress predicted by the model is consistent with that measured by LoCKNER (1998) up to $P_{c}=400 \mathrm{MPa}$, above which stress quartz reaches its flow threshold and the entire granite sample deforms by plastic flow.

Having found values of $E_{1}=96 \mathrm{GPa}$ and $E_{2}=61.3 \mathrm{GPa}$ that are consistent with the observed values of the failure stress and with the total nonlinear strain at failure when $P_{c}=-200 \mathrm{MPa}$, we now compare the nonlinear stress-strain curve predicted by the bi-mineral model with that measured by LOCKNER (1998). The theoretical stress-strain curve is generated by loading the model in Fig. 9 by a sequence of equal strain increments $\Delta \epsilon_{o}=\dot{\epsilon}_{o} \Delta t$, where $\dot{\epsilon}_{o}$ is the given axial loading rate in the triaxial 

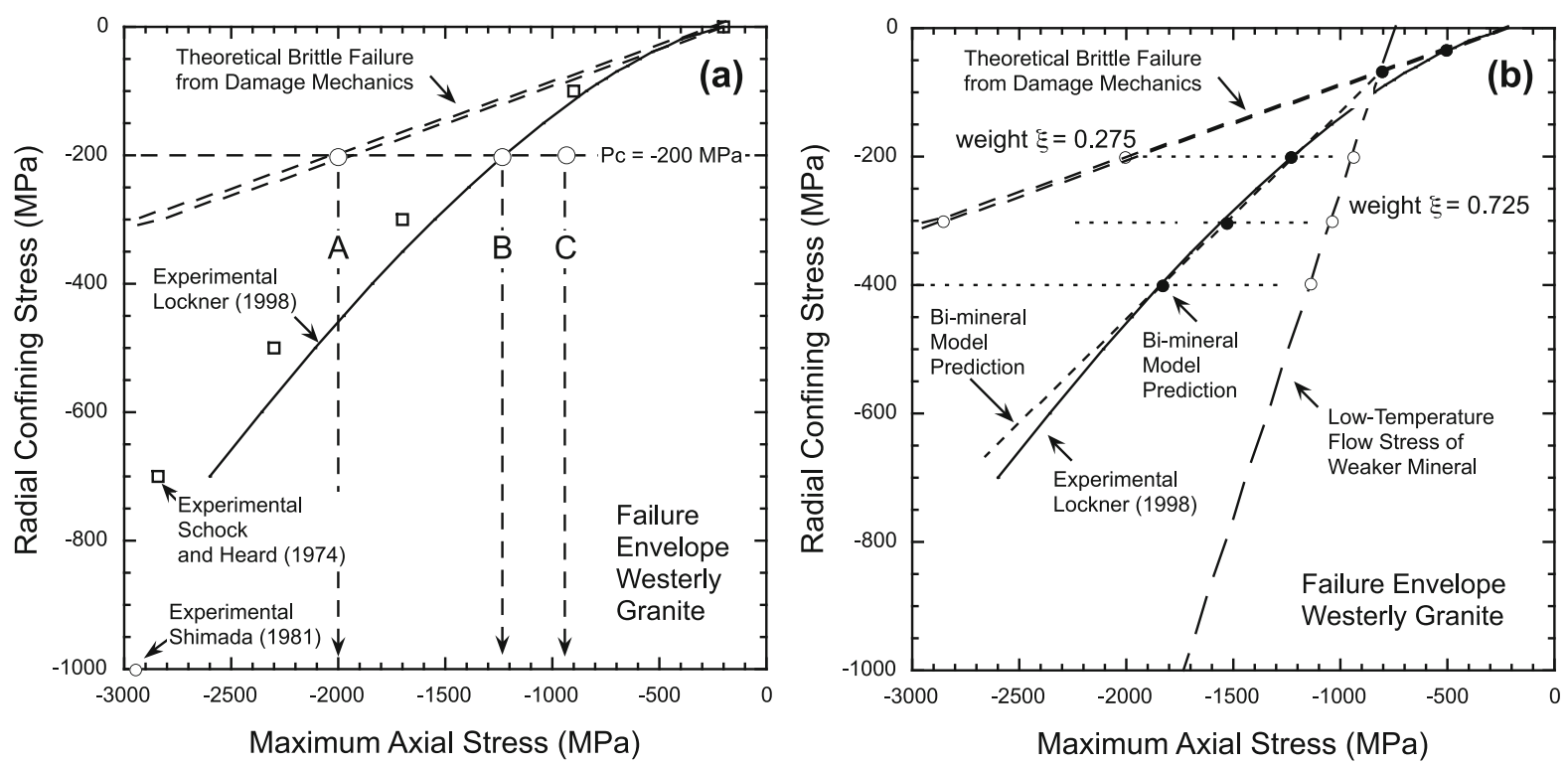

Figure 11

Failure envelope for Westerly granite based on the bimineralic model and compared with data

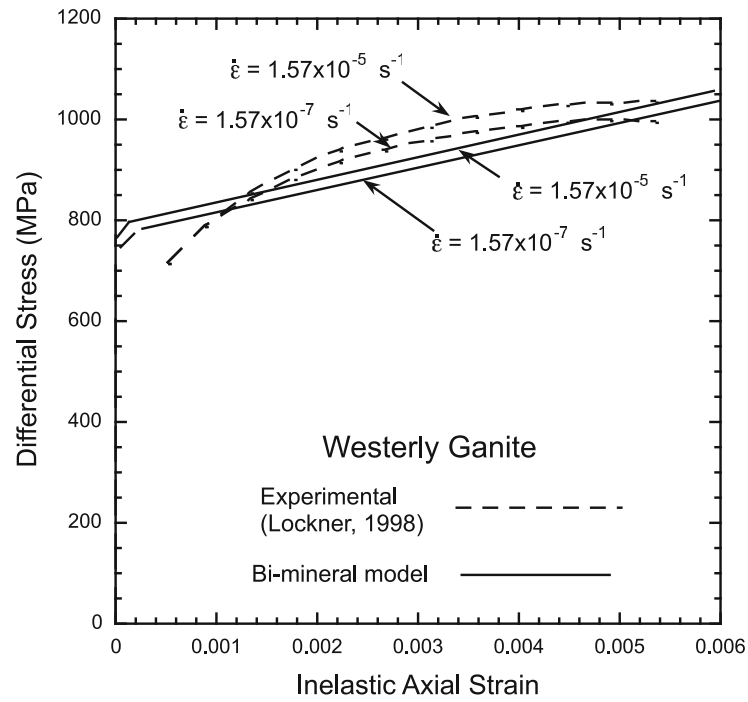

Figure 12

Differential stress versus inelastic strain for Westerly granite based on the bimineralic model and compared with experimental data

experiment. Each strain increment produces an instantaneous increment of stress $\Delta \sigma_{i}=E_{i} \Delta \epsilon_{o}$ (where $i=1$ or 2 for quartz or feldspar respectively). In the feldspar, Eq. 48 is used to relax the strain over the time increment by an amount $\Delta \epsilon_{2 n l}=\dot{\epsilon}_{2 n l} \Delta t$, where $\dot{\epsilon}_{2 n l}$ depends on the total shear stress in the feldspars $\left(\sigma_{2}-P_{c}\right)$ and the flow parameters in
Table 1. The stress $\sigma_{2}$ in the feldspars is then reduced by $\Delta \sigma_{2}=E_{2} \Delta \epsilon_{2 n l}$. The macroscopic stress $\bar{\sigma}$ at that time is then calculated according to Eq. 46 before the next strain increment is applied.

The nonlinear axial strain is calculated as the difference between the strain found by the model at $\bar{\sigma}$ and the purely elastic strain $\epsilon_{e l}=\bar{\sigma} /\left[\xi E_{1}+(1-\right.$ $\left.\xi) E_{2}\right]$ that would have accumulated at that stress if the deformation were totally linear. Model values of nonlinear strain are plotted in Fig. 12 where they are compared with measurements from LOCKNER (1998). Note that the magnitude of the model strain at failure and its general increase with differential stress are in rough agreement with the data, but the slope predicted by the model is constant while the data has significant curvature. The constant slope predicted by the model is easily understood. The stress in the quartz rises linearly throughout loading until it reaches the brittle failure level. However, although the rise in stress in the feldspar is initially linear, it slows and stops at the stress $\sigma_{s 2}$ for which $\dot{\epsilon}_{2 n l}$ in Eq. 48 is equal to the loading rate $\dot{\epsilon}_{o}$. Since the entire increase in $\sigma_{2}$ due to loading is relaxed by flow, $\sigma_{2}$ never rises above this level. The slope of the stress strain curve above this point is again linear $\Delta \bar{\sigma} / \Delta \epsilon=\xi E_{1}$, and therefore, the difference between this strain and the pure elastic strain is also linear. 
The stress level in the feldspars at which the loading rate is equal to the flow stress increases at $2.8 \%$ per order of magnitude increase in loading rate as in Fig. 12. Since the feldspars support $72.5 \%$ of the load, the result is an increase in the failure stress of about $2 \%$ for every order of magnitude increase in the loading rate - in agreement with the increases in strength with increasing loading rate reported by LOCKNER (1998). The failure of the model to capture the curvature in the data may be due to the fact that dislocation glide has a long transient before the steady state (assumed here) is achieved AshBY and VerRall (1977). It may also be due to a contribution from the damage, which would produce negative curvature by adding a bit of extra strain near failure.

\subsection{Effect of Heterogeneity}

Figure $11 \mathrm{~b}$ shows a fit of the bi-mineral model to the entire failure envelope for Westerly granite. The quartz is brittle up to a confining stress of about $7.8 \mathrm{GPa}$, above which it also yields. The problem with this interpretation is that it requires the yield strength of quartz to be nearly ten times larger than that of feldspar, which is not supported by the smaller difference in their hardness. Nor is it supported by the more linear failure envelope for aplite, another quartz-feldspar rock (Fig. 6), which can be fit using a much smaller difference in yield stress for the two minerals.

This apparent contradiction can be resolved if granite is more mechanically heterogeneous than is aplite. If we allow a range of both flow strength and local stress, then the larger and smaller yield surfaces in the case of granite are only apparent and represent limits of high stress and low yield strength on the one hand and low stress and high yield strength on the other. The flow parameters in Table 1 reflect this artificially large range, particularly the lattice resistance $\hat{\tau}_{p} / G$. The real values are probably closer to the mean.

\section{Discussion and Conclusions}

The micromechanical damage model originally formulated by AshBY and SAmmis (1990) makes several simplifying assumptions. All cracks are assumed to be the same size and parallel and they are assumed to interact in a globally average sort of way. In spite of this simplicity, the model gives a reasonable description of the failure surface for a wide variety of rocks at low to intermediate confining pressure and at the low loading rates typical in triaxial laboratory experiments (AshBY and SAmmis, 1990; BAud et al., 2000a, b) . It does not, however, simulate the observed curvature in either the stressstrain curve near failure or in the failure surface at high confining pressures. We have shown here that the extension of the Ashby and SAmmis (1990) model to allow a range of flaw sizes and orientations does not produce significant curvature. However, the observed nonlinearities can be simulated if granite is modeled as a bi-mineralic rock where one of the minerals (feldspar) is allowed to creep according to a high-stress dislocation glide mechanism. This bimineral model quantifies the assertion in AsHBY and SAmmis (1990) that the curvature in the failure surface is due to a broad transition from brittle to ductile mechanisms.

It is interesting to compare the results here with a similar study that fits the triaxial data for Westerly granite using a continuum damage mechanics model (LyakHOvsky et al., 1997; 2005; HAMiel et al., 2004). In this model, damage is quantified by a scalar parameter $\alpha$, which is assumed to evolve according to

$$
\frac{d \alpha}{d t}= \begin{cases}C_{d}\left(\xi-\xi_{o}\right) I_{2} & \xi \geq \xi_{o} \\ C_{1} \exp \left(\alpha / C_{2}\right)\left(\xi-\xi_{o}\right) I_{2} & \xi<\xi_{o}\end{cases}
$$

where $I_{1}, I_{2}$ are the first and the second strain invariants, respectively, $\xi=I_{1} / \sqrt{I_{2}} \cdot C_{1}, C_{2}$ and $C_{d}$ are empirical constants fit to laboratory data (LYAKHOVSKY et al., 1997, 2005). In order to fit the triaxial failure data for granite the authors made $C_{d}$ an empirical function of the confining pressure.

The effective elastic behavior is given by

$$
\sigma_{i j}=\frac{\partial W}{\partial \epsilon_{i j}}
$$

where an additional second order term strain term is added to the elastic energy density where $\gamma$ is a new elastic constant that adjusts the stress-strain behavior for the damage. It is assumed that the first Lame 
constant is not affected by the damage $\left(\lambda=\lambda_{o}\right)$, but that the shear modulus and new elastic constant depend on the damage as

$$
\mu=\mu_{o}+\alpha^{\beta} \quad \gamma=\gamma_{o}+\alpha^{\beta}
$$

where $\beta$ is another empirical constant that is adjusted to fit the curvature in the stress-strain curve.

The difference between the continuum and micromechanical formulations of damage mechanics becomes apparent when modeling phenomena that involve high loading rates such as the process zone at the tip of an earthquake rupture, a meteorite impact, or an underground explosion. The continuum formulation assumes that the empirical rate Eq. 49 also holds at high loading rates. However, since the micromechanical model incorporates damage mechanics, it can naturally include theoretical and experimental results on high-speed fracture propagation which modify the critical stress intensity factor for the nucleation of the wing cracks and relate their propagation velocity to the difference between the instantaneous and equilibrium stress intensity factors (LiU and Rosakis, 1994; LiU et al., 1998; Rosakis and Zehnder, 1985; Owen et al., 1998; ZeHNDER and Rosakis, 1990).

Since the micromechanical model separates out brittle and ductile deformation mechanisms, it allows a more physical extrapolation of each mechanism to very high loading rates where the ductile mechanisms can be ignored. In the end, the formulation that gives an better representation of deformation at very high loading rates can only be determined by comparison with experimental data.

\section{Acknowledgments}

The authors would like to thank Yehuda Ben-Zion and three anonymous reviewers for their constructive reviews. This research was funded through the National Science Foundation collaborative grant EAR-0711171 to the University of Southern California and the California Institute of Technology, the National Science Foundation for the research grant (award no. EAR-0911723), provided under the American Recovery and Reinvestment Act of
2009 (ARRA) (Public Law 111-5) and the Department of the Air Force though Grant \#FA8718-08C-0026.

\section{Appendix A: Calculating the Strain Energy and Axial Strain Due to Sliding on the Initial Inclined Cracks}

The average value of the stress intensity factor, $K$ around the edge of the crack in Eq. 6 is

$$
\begin{aligned}
\left\langle K_{I I}^{2}+K_{I I I}^{2}\right\rangle= & \frac{32 a \sigma_{s}^{2}}{\pi^{2}(2-v)^{2}} \\
& \times \int_{0}^{\pi / 2}\left[\cos ^{2} \phi+(1-v)^{2} \sin ^{2} \phi\right] d \phi \\
& =\frac{8 a \sigma_{s}^{2}}{\pi}\left[\frac{1+(1-v)^{2}}{(2-v)^{2}}\right]
\end{aligned}
$$

and Eq. 6 becomes

$$
\begin{aligned}
\Delta W_{1} & =\frac{16 \sigma_{s}^{2}}{E_{o}}\left[\frac{1+(1-v)^{2}}{(2-v)^{2}}\right] \int_{0}^{a} r^{2} d r \\
& =\frac{16 a^{3} \sigma_{s}^{2}}{3 E_{o}}\left[\frac{1+(1-v)^{2}}{(2-v)^{2}}\right]
\end{aligned}
$$

The uniaxial strain $\epsilon_{1}$ can be calculated as (recall that $\left.\sigma_{s}=\tau+\mu \sigma_{n}\right)$

$$
\begin{aligned}
& \epsilon_{1}= \frac{\partial W}{\partial \sigma_{1}}=\frac{\sigma_{1}}{E_{o}}+ \\
& N_{V}\left(\frac{\partial \Delta W_{1}}{\partial \sigma_{s}}\right)\left(\frac{\partial \sigma_{s}}{\sigma_{1}}\right) \\
&=\frac{\sigma_{1}}{E_{o}}+ \\
& N_{V}\left(\frac{\partial \Delta W_{1}}{\partial \sigma_{s}}\right)\left(\frac{\partial \tau}{\partial \sigma_{1}}+\mu \frac{\partial \sigma_{n}}{\partial \sigma_{1}}\right) \\
&=\frac{\sigma_{1}}{E_{o}}+N_{V}\left(\frac{\partial \Delta W_{1}}{\partial \sigma_{s}}\right)\left(\frac{1+\mu}{2}\right) \\
& \Rightarrow \epsilon_{1}=\frac{\sigma_{1}}{E_{o}}-\frac{32 N_{V} a^{3} \sigma_{s}}{6 E_{o}}(1+\mu) \\
& \times\left[\frac{1+(1-v)^{2}}{(2-v)^{2}}\right]
\end{aligned}
$$


In terms of the principal stresses

$$
\begin{aligned}
\epsilon_{1}= & \frac{\sigma_{1}}{E_{o}}+\frac{2 D_{o}}{\pi \alpha^{3} E_{o}}(1-\mu)^{2} \\
& \times\left[\frac{1+(1-v)^{2}}{(2-v)^{2}}\right] \\
& \times\left[\sigma_{1}-\frac{(1+\mu)}{(1-\mu)} \sigma_{3}\right]
\end{aligned}
$$

\section{Appendix B: Estimation of Nonlinear Strain}

\section{From the Moment Tensors}

A second estimate of the strain associated with the damage can be obtained by summing the seismic moments of the individual flaws. Kostrov (1974) gives the following expression for the macroscopic strain in a volume $V$ containing $n$ flaws

$$
\epsilon_{i j}=\frac{1}{\mu V} \sum_{n=1}^{N} M_{i j}
$$

Johnson and SAmmis (2001) express the scalar moment density associated with an individual angle crack and its wing cracks as a shear moment associated with sliding on the angle crack and a tensile moment associated with the opening of the wing cracks.

$$
m=m_{s}+m_{t}
$$

where

$$
\begin{gathered}
m_{s}=\frac{9}{2}\left(\frac{\lambda+2 G}{\lambda+G}\right) \frac{D_{o} K_{I C}}{\sqrt{\pi a} \cos ^{5 / 2} \Psi \sin \Psi} \\
\times\left[\left(\frac{D}{D_{o}}\right)^{1 / 3}-1\right]^{1 / 2} \\
m_{t}=\frac{3}{2} \frac{(\lambda+2 G)^{2}}{G(\lambda+G)} \frac{D_{o} K_{I C}}{\sqrt{\pi a \cos \Psi}} \times\left[\left(\frac{D}{D_{o}}\right)^{1 / 3}-1\right]^{5 / 2} \\
\frac{m_{t}}{m_{s}}=\frac{\lambda+2 G}{3 G} \sin \Psi \cos ^{2} \Psi \times\left[\left(\frac{D}{D_{o}}\right)^{1 / 3}-1\right]^{2}
\end{gathered}
$$

In these expressions Johnson and SAmmis (2001) ignore the $\beta$ factor in AsHBY and SAmmis (1990) since it gives a contribution to the moment at zero stress.
Note that the tensile opening of the wing cracks makes an increasingly dominant contribution as the damage increases.

For a crack opening in the $x_{2}$ direction, the moment density tensor is

$$
\mathbf{m}=m_{s}\left[\begin{array}{ccc}
-1 & 0 & 0 \\
0 & 1 & 0 \\
0 & 0 & 0
\end{array}\right]+m_{t}\left[\begin{array}{ccc}
\zeta & 0 & 0 \\
0 & 1 & 0 \\
0 & 0 & \zeta
\end{array}\right]
$$

where $\zeta=\frac{\lambda}{\lambda+\mu}=1-\frac{c_{s}^{2}}{c_{p}^{2}}$. Here $c_{s}$ and $c_{p}$ are the S- and the P-wave speeds, respectively.

Kostrov's expression for the axial strain is

$$
\epsilon_{i j}=\frac{1}{\mu V} \sum_{n=1}^{N} M_{i j}=\frac{1}{\mu}\left(m_{s}+\zeta m_{t}\right)
$$

For $\Psi=45^{\circ}$ we have

$$
\begin{aligned}
\epsilon_{11}= & \frac{3}{2^{3 / 4}}\left(\frac{\lambda+2 G}{\lambda+G}\right) \\
& \times \frac{K_{I C} D_{o}}{G \sqrt{\pi a}}\left[\left(\frac{D}{D_{o}}\right)^{2 / 3}-1\right]^{1 / 2} \\
& \times\left\{\frac{12}{\sqrt{2}}+\frac{\lambda}{G}\left[\left(\frac{D}{D_{o}}\right)^{2 / 3}-1\right]^{2}\right\}
\end{aligned}
$$

The first term in the curly brackets is associated with sliding on the initial angle cracks while the second term is associated with tensile opening of the wing cracks.

\section{REFERENCES}

Ashby, M., and S. D. Hallam Née Cooksley (1986), The failure of brittle solids containing small cracks under compressive stress states, Acta Metall. Mater., 34(3), 497-510.

Ashby, M. F., and C. G. Sammis (1990), The damage mechanics of brittle solids in compression, Pure Appl. Geophys., 133(3), 489-521.

Ashby, M. F., and R. A. Verrall (1977), Micromechanisms of flow and fracture, and their relevance to the rheology of the upper mantle, Phil. Trans. R. Soc. Lond. A, 288, 59-95.

BAss, J. (1995), Elasticity of minerals, glasses, and melts, in Mineral Physics and Crystallography: A Handbook of Physical Constants, vol. 2, edited by T. J. Ahrens, pp. 45-63, American Geophysical Union.

Baud, P., A. Schubnel, and T. Wong (2000a), Dilatancy, compaction, and failure mode in Solnhofen limestone, J. Geophys. Res., 105(B8), 19,289-19,303.

Baud, P., W. Zhu, and T. Wong (2000b), Failure mode and weakening effect of water on sandstone, J. Geophys. Res., 105(B7), 16,371-16,389. 
Brace, W. F. (1965), Some new measurements of linear compressibility of rocks, J. Geophys. Res., 70(2), 391-398.

BYERLEE, J. (1967), Frictional characteristics of granite under high confining pressure, J. Geophys. Res., 72(14), 3639-3648.

Deshrande, V. S., and A. G. Evans (2008), Inelastic deformation and energy dissipation in ceramics: A mechanism-based constitutive model, J. Mech. Phys. Solids, 56(10), 3077-3100.

Hadley, K. (1976), Comparison of calculated and observed crack densities and seismic velocities in westerly granite, J. Geophys. Res., 81(20), 3484-3494.

Hamiel, Y., Y. Liu, V. Lyakhovsky, Y. Ben-Zion, and D. Lockner (2004), A viscoelastic damage model with applications to stable and unstable fracturing, Geophys. J. Int., 159(3), 1155-1165.

Huffman, A., J. Brown, N. Carter, and W. Reimold (1993), The microstructural response of quartz and feldspar under shock loading at variable temperatures, J. Geophys. Res., 98(B12), 22,171-22,197.

Jeyakumaran, M., and J. W. RUdNicki (1995), The sliding wing crack-again, Geophys. Res. Lett., 22(21), 2901-2904.

Johnson, L. R., and C. G. SAmmis (2001), Effects of rock damage on seismic waves generated by explosions, Pure Appl. Geophys., 158(11), 1869-1908.

Kachanov, M. L. (1982a), A microcrack model of rock inelasticity part I: Frictional sliding on microcracks, Mech. Mater., 1(1), 19-27.

KaCHANOv, M. L. (1982b), A microcrack model of rock inelasticity part II: Propagation of microcracks, Mech. Mater., 1(1), 19-27.

Kostrov, B. V. (1974), Seismic moment and energy of earthquakes, and seismic flow of rock, Izv. Acad. Sci. USSR Phys. Solid Earth, $1,23-44$.

Liu, C., and A. J. Rosakis (1994), Investigation of transient effects for dynamically initiating and growing cracks under stress wave loading conditions, in Dynamic Fracture Mechanics, edited by M. H. Aliabadi, chap. 4, Computational Mechanics Publication, Southhampton, UK; Boston, USA.

Liu, C., W. G. Knauss, and A. J. Rosakis (1998), Loading rates and the dynamic initiation toughness in brittle solids, Int. J. Fract., 90(1), 103-118.
LOCKNER, D. (1998), A generalized law for brittle deformation of westerly granite, J. Geophys. Res., 103(B3), 5107-5123.

Lyakhovsky, V., Y. Ben-Zion, and A. Agnon (1997), Distributed damage, faulting, and friction, J. Geophys. Res., 102(27), 635-27.

Lyakhovsky, V., Y. Ben-Zion, and A. Agnon (2005), A viscoelastic damage rheology and rate- and state-dependent friction, Geophys. J. Int., 161(1), 179-190.

Nemat-Nasser, S., and H. HoriI (1982), Compression-induced nonplanar crack extension with application to splitting, exfoliation, and rockburst, J. Geophys. Res., 87(B8), 6805-6821.

O'Connell, R. J., and B. Budiansky (1974), Seismic velocities in dry and saturated cracked solids, J. Geophys. Res., 79(35), 5412-5426.

Owen, D. M., S. Zhuang, A. J. Rosakis, and G. Ravichandran (1998), Experimental determination of dynamic crack initiation and propagation fracture toughness in thin aluminum sheets, Int. J. Fract., 90(1), 153-174.

Rosakis, A., and A. Zehnder (1985), On the dynamic fracture of structural metals, Int. J. Fract., 27(3), 169-186.

SCHOCK, R., and H. HEARD (1974), Static mechanical properties and shock loading response of granite, J. Geophys. Res., 79, 1662-1666.

Shimada, M. (1981), The method of compression test under high pressures in a cubic press and the strength of granite, Tectonophysics, 72(3-4), 343-357.

TADA, H., P. C. PARIS, and G. R. IRWIN (1985), The stress analysis of cracks handbook, Paris Productions (and Del Research Corp.) St. Louis, MO.

WAWERSIK, W. (1973), Time-dependent behavior of rock in uniaxial compression, in Proc. 14th Symposium on Rock Mechanics, pp. 85-106.

Zehnder, A. T., and A. J. Rosakis (1990), Dynamic fracture initiation and propagation in 4340 steel under impact loading, Int. J. Fract., 43(4), 271-285.

(Received July 1, 2010, revised December 30, 2010, accepted December 31, 2010) 